\title{
Three-dimensional conformal radiotherapy by delineations on CT-based simulation in different respiratory phases for the treatment of senile patients with non-small cell lung cancer
}

\author{
This article was published in the following Dove Press journal: \\ OncoTargets and Therapy \\ 7 September 2015 \\ Number of times this article has been viewed
}

\section{Weifeng Wang ${ }^{1,2}$ \\ Feng Yuan ${ }^{2}$ \\ Guoping Wang ${ }^{2}$ \\ Zhiren Lin ${ }^{2}$ \\ Yanling Pan ${ }^{2}$ \\ Longhua Chen'}

'Department of Radiation Oncology, Nanfang Hospital, Southern Medical University, Guangzhou, Guangdong Province, People's Republic of China; ${ }^{2}$ Department of Radiation Oncology, Haikou municipal hospital, Haikou, Hainan Province, People's Republic of China

Correspondence: Longhua Chen Department of Radiation Oncology, Nanfang Hospital, Southern Medical University, Guangzhou 5105I5,

Guangdong Province, People's Republic of China

Tel +86 I520366 8867

Fax +86 2I 64085875

Email chenlonghuasci@।26.com
Aim: This study aimed to evaluate the application of three-dimensional conformal radiotherapy (3D-CRT) for elderly patients with non-small cell lung cancer (NSCLC) based on computed tomography $(\mathrm{CT})$ simulations in different respiratory phases.

Methods: A total of 64 patients aged $>70$ years old with NSCLC were treated by 3D-CRT using CT images in different respiratory phases. The gross tumor volumes (GTVs) at the end of inspiration and end of expiration were combined to obtain the total GTV, which was close to the motional range of tumors during respiration, and no additional expansion of the clinical target volume (CTAV) to planning target volume (PTV) (CTAV:PTV) was included during the recording of respiratory movements. Patients were also planned according to the classic 3D-CRT approach. Efficacy, prognostic factors, and side effects were evaluated.

Results: Compared with the classic approach, the average PTV was $18.9 \%$ lower (median: $17.3 \%$ ), and the average lung volume receiving a prescribed dose for a tumor was $22.4 \%$ lower (median: 20.9\%). The 1-, 2-, and 3-year survival rates were $70.6 \%, 54.9 \%$, and $29.4 \%$, respectively, with an overall tumor response rate of $79.7 \%$. The Karnofsky performance status and $\mathrm{N}$ stage were independent prognostic factors, whereas age was not.

Conclusion: Without affecting therapeutic effects, CT simulations in different respiratory phases were well-tolerated in elderly patients with NSCLC, could effectively reduce PTV, and could improve the quality of life.

Keywords: non-small cell lung cancer, three-dimensional conformal radiotherapy, CT-based simulation in different respiratory phases, survival

\section{Introduction}

Lung cancer is the deadliest form of cancer worldwide. ${ }^{1}$ Indeed, approximately $80 \%$ of lung cancer patients present with an advanced stage of the disease (Stages III and IV) and are considered inoperable due to locoregional tumor extension, extrathoracic spread, or elderly age with poor physical condition. ${ }^{2}$ For these patients, multidisciplinary treatments are often necessary. However, elderly patients often suffer from comorbidities, and thus surgery, conventional radiotherapy, and chemotherapy are risky for them.

Three-dimensional conformal radiotherapy (3D-CRT) is a new modality of radiotherapy that has been designed for patients with inoperable disease or who refused surgery. ${ }^{3}$ A meta-analysis has shown that 3D-CRT improved local control rates compared with conventional radiotherapy. ${ }^{3}$ However, 3D-CRT is associated with some 
disadvantages. Indeed, treatment sessions are longer, introducing the problem of motion during treatment, particularly in elderly patients with comorbidities. ${ }^{4}$ Therefore, there is a need for improved strategies that could cope with these motions, particularly respiratory motions.

Different strategies were evaluated to improve 3D-CRT treatments, including volumetric modulated arc therapy, ${ }^{4}$ respiratory-gated 3D-CRT, ${ }^{5}$ and different methods of compensation for pulmonary motions (free breathing, breath holding, abdominal compression, etc). ${ }^{6}$ Planning using respiration-correlated computed tomography (CT) allows the assessment of patient-specific pulmonary motions of the tumor and has been suggested to be reliable. ${ }^{7}$ This new approach could allow smaller irradiation fields that could spare more normal lung tissue.

Therefore, the purpose of the present study was to verify that CT simulations in different respiratory phases before 3D-CRT may reduce the irradiated lung volume. To do so, we analyzed 64 patients with non-small cell lung cancer (NSCLC) who accepted to undergo planned 3D-CRT using CT simulations in different respiratory phases and a 4-year follow-up. Treatment outcomes and prognosis factors were analyzed.

\section{Materials and methods Patients}

Sixty-four patients aged 70-88 years and newly diagnosed with NSCLC (pathologically confirmed) were enrolled at the Haikou People's Hospital from September 2009 to June 2013. All 64 patients were considered to be medically inoperable based on the following conditions: poor pulmonary function in 25 , cardiac disease in 28 , poor general condition in seven, or other medical conditions in four patients. Exclusion criteria were severe pulmonary hypofunction or $<3$ months of expected survival.

Baseline assessments included a medical history, physical examination, complete blood count, comprehensive blood chemistry evaluation, creatinine clearance, chest X-ray, and CT scan of the chest and upper abdomen or a positron emission tomography-CT scan. Bone scans and brain imaging were performed only if clinically indicated. This study was approved by the local Medical Ethics Committee, and written informed consent was obtained from all patients.

\section{End points}

The primary end point was objective response rate of target lesions. Secondary end points included toxicity, overall survival (OS), and median OS time.

\section{Treatments}

Breathing training to ensure the repeatability of positioning and treatment of orthostatic position Thermoplastic membrane fixed shelves (CIVCO Medical Solutions, Kalona, IA, USA) were used. The magnitude of respiration was measured, and quiet and hold respirations were observed and recorded using a simulator machine to ensure that the patient could accomplish end-stage exhalation and inhalation under the instructions of the doctor (respiration or target motions included the following: up and down, front and back, and the left and right directions). A CT enhancement scan with $5 \mathrm{~mm}$ thickness in two different phases (end inhalation in the arterial phase and end exhalation in the venous phase) was performed for each patient, and the CT data were then sent to a treatment planning system station.

\section{Target delineation and treatment planning}

Contour delineation and respiratory motion validation

The magnitude of respiratory and target motion was determined by comparing the center of the gross tumor volume (GTV) in the end-exhalation and end-inhalation phases.

\section{Target definition}

The gross tumor and metastatic lymph nodes (diameter $\geq 10 \mathrm{~mm}$ ) were defined as GTV: GTV (in the arterial phase) and $\mathrm{GTV}_{\mathrm{v}}$ (in the venous phase). The magnitude of the GTV motion was determined by the addition of $\mathrm{GTV}_{\mathrm{A}}$ to $\mathrm{GTV}_{\mathrm{v}}$ $\left(\mathrm{GTV}_{\mathrm{A}}+\mathrm{GTV}_{\mathrm{v}}\right)$, which was defined as the full-time phase, and expansion of the GTV with a uniform margin in all directions was defined as the clinical tumor volume (CTUV). The CTUV was expanded in the $8 \mathrm{~mm}$ range for adenocarcinoma, in the $6 \mathrm{~mm}$ range for squamous cell carcinoma, ${ }^{8}$ and $15 \mathrm{~mm}$ away from the main bronchus line for central lung cancers near the main bronchus. The CTUV was then equal to the internal target volume. The planning target volume (PTV) was obtained from the internal target volume with a $5 \mathrm{~mm}$ expansion.

\section{Radiation field design}

Setting five to six coplanar or non-coplanar conformal fields is an optimal dose distribution in the dose--volume histogram, and the PTV was completely included with a $90 \%$ dose. The V20 of the lungs was $<25 \%$, and the dose for the spinal cord and the esophagus was ensured as being safe.

\section{Dose fractionation regimen}

A single dose of 2-3.6 Gy repeated 17-34 times was used for primary lung tumors, and the GTV received a total dose of 61.2-72 Gy (equivalent dose was 68-81.6 Gy, $\alpha / \beta=10$ ). 


\section{Breathing}

The patients maintained quiet breathing during treatment in a CT simulation position.

\section{Comparison of target delineation with the classic 3D-RCT}

Target delineation procedure was performed in all 64 patients using conventional $\mathrm{CT}$ and using $\mathrm{CT}$ images in different respiratory phases before therapy. The GTV was based on the quiet inspiratory breathing phase. The field was set as mentioned in this study, but the PTV margin was applied to the CTUV to account for potential target motion and setup errors according to the conventional method. Then, a comparison to the volume of lungs with a prescribed dose and PTV of the two methods of target delineation was performed.

\section{Follow-up and response evaluation}

All patients completed the treatment regimen and had a follow-up 1 month later, followed by a follow-up every 3 months up to 3 years and then every 6 months during the fourth to fifth years of follow-up. Tumor response and short-term efficacy evaluations were performed according to World Health Organization reporting standards (Response Evaluation Criteria In Solid Tumors [RECIST]), ${ }^{8}$ and longterm efficacy was assessed by the 1- to 3-year OS. Complete response (CR) was defined as the disappearance of all target lesions. Partial response (PR) was defined as $>30 \%$ decrease in the sum of the longest diameter of target lesions, taking the baseline sum of the longest diameter as reference. Progressive disease (PD) was defined as $>20 \%$ increase in the sum of the longest diameter of target lesions, taking the smallest sum of the longest diameter recorded since the treatment began or the appearance of one or more new lesions as reference. Stable disease was defined as neither sufficient shrinkage to qualify as PR nor a sufficient increase to qualify as PD, taking the smallest sum of the longest diameter since the treatment began. Toxicities were assessed according to the Radiation Therapy Oncology Group (RTOG) score. ${ }^{8}$

\section{Statistical analysis}

Statistical analysis was performed using SPSS 13.0 (SPSS Inc, Chicago, IL, USA). Survival was calculated using the Kaplan-Meier method, and curves were compared using the log-rank method. Categorical variables are presented using proportions and were analyzed using the chi-square test. Cox regression analysis was used to reveal the influencing factors of therapy outcomes. Two-sided $P$-values $<0.05$ were considered statistically significant.

\section{Results}

\section{Baseline characteristics of the patients}

The baseline characteristics of the 64 patients who accepted the new 3D-CRT approach and the 4-year follow-up are shown in Table 1. Median age was 76 years (range: $70-84$ years) in 41 men (64.1\%) and 23 women (35.9\%). Most patients were diagnosed with adenocarcinoma $(50.0 \%)$ or squamous carcinoma (39.0\%) by histology examination $(79.6 \%)$. The Karnofsky performance status (KPS) was 100 in two patients (3.1\%), 90 in six (9.4\%), 80 in 28 (43.8\%), 70 in 21 (32.8\%), and $<60$ in $7(10.9 \%)$. Most patients presented with Stage IIIa $(n=25,39.1 \%)$ or IIIb $(n=21,32.8 \%)$ disease.

\section{Comparison with the classic 3D-RCT}

Compared with the classic approach, a reduced average PTV of $18.9 \%$ (median of $17.3 \%$ ) was obtained using the new approach, and the average volume of normal lung receiving radiations was $230 \mathrm{~cm}^{3}$ in the new approach and $293 \mathrm{~cm}^{3}$ in the classic approach (Figure 1). The respiratory phase positioning method reduced the irradiated volume by an average of $22.4 \%$ (median: $20.9 \%$ ).

Table I Baseline characteristics of the 64 patients

\begin{tabular}{ll}
\hline Characteristics & $\mathbf{N}(\%)$ \\
\hline Sex & \\
Male & $4 \mathrm{I}(64 . \mathrm{I})$ \\
Female & $23(35.9)$ \\
Diagnosis & \\
Cytology & $9(14.1)$ \\
Histology & $51(79.6)$ \\
Unknown & $4(6.3)$ \\
Histologic subtype & \\
Adenocarcinoma & $32(50.0)$ \\
Squamous carcinoma & $25(39.0)$ \\
Mixed carcinoma & $3(4.7)$ \\
Unknown & $4(6.3)$ \\
Karnofsky performance status & \\
I00 & $2(3.1)$ \\
90 & $6(9.4)$ \\
80 & $28(43.8)$ \\
70 & $21(32.8)$ \\
S60 & $7(10.9)$ \\
Disease stage at diagnosis & \\
Ib & $3(4.7)$ \\
Ila & $4(6.3)$ \\
Ilb & $7(10.9)$ \\
Illa & $25(39.1)$ \\
Illb & $21(32.8)$ \\
Age (years) & $4(6.3)$ \\
Median & \\
Range & 76 \\
\hline & $70-84$ \\
\hline
\end{tabular}




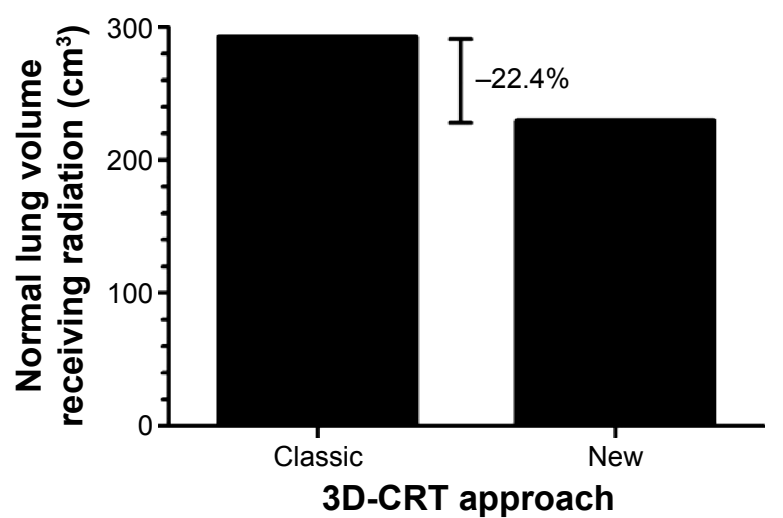

Figure I Comparison of normal lung volume receiving radiation between the classic and the new 3D-CRT approaches.

Abbreviation: 3D-CRT, three-dimensional conformal radiotherapy.

\section{Evaluation of short-term efficacy}

All patients completed their treatment regimens, and the CR rate was 26.6\% (17/64), PR was 53.1\% (34/64), and odds ratio was $79.7 \%(51 / 64)$. Moreover, chest pain symptoms completely disappeared in seven of eight cases after treatment, and one case was able to live without painkillers. Radiation pneumonitis occurred in five out of $64(7.8 \%)$ patients, including a patient with RTOG Grade 3 disease who obtained symptomatic relief after treatment. Esophagitis occurred in six out of $51(11.8 \%)$ patients who were of RTOG Grade 2 or less (Table 2).

\section{Long-term efficacy and follow-up}

Follow-up lasted at least 30 months for all patients, for a median of 42 months. Thirteen patients died from nontumor-related diseases and natural death. The median OS was 27 months, and the 1-, 2-, and 3-year OS rates were 70.6\%, $54.9 \%$, and $29.4 \%$, respectively (Figure 2A).

Using the median age for grouping (76 years), the OS difference was not significant $(P=0.84)$ (Figure 2B). Comparison of KPS scores $<80$ and $\geq 80$ demonstrated that the OS difference was statistically significant $(P=0.016)$ (Figure 2C). Comparison of the initial tumor stage for patients who were $>$ T2 and $\leq$ T2 showed no significant difference

Table 2 Evaluation of short-term efficacy and toxicity of the radiotherapy

\begin{tabular}{ll}
\hline Criteria & $\mathbf{N}$ (\%) \\
\hline Complete response & $17 / 64(26.6)$ \\
Partial response & $34 / 64(53.1)$ \\
Overall response rate & $51 / 64(79.7)$ \\
Chest pain completely disappeared & $7 / 8(87.5)$ \\
Pneumonitis & $5 / 64(7.8)$ \\
Esophagitis & $6 / 51(11.8)$ \\
\hline
\end{tabular}

in $\mathrm{OS}(P=0.988)$ (Figure 2D). For patients with lymph node staging $>\mathrm{N} 1$ and $\leq \mathrm{N} 1$, the OS difference was statistically significant $(P=0.018)$ (Figure $2 \mathrm{E})$.

\section{Multivariate analysis}

Cox regression analysis was performed. Variables including age, $\mathrm{T}$ stage, $\mathrm{N}$ stage, and KPS score were entered into the regression equation. As shown in Table 3, N stage and KPS scores were independent factors associated with survival.

\section{Discussion}

Most elderly patients with NSCLC may exhibit limited tolerance to surgery, chemotherapy, and conventional radiotherapy because of comorbidities such as cardiovascular disease and chronic pulmonary diseases, or physical feebleness. In the past 10 years, mortality due to NSCLC in patients $<50$ years old declined, but this increased in patients $>70$ years old. Therefore, oncologists must determine adequate treatment strategies that are tailored to this specific population of patients. For elderly patients, surgery remains the first choice for early-stage NSCLC and can achieve similar efficacy as in younger patients based on well-designed and safe surgery, but radiation therapy is the optimal choice for those who are inoperable or refuse surgery. ${ }^{9}$ The side effects of conventional radiotherapy for inoperable end-stage patients are increased due to excessively large exposure volume. Therefore, new strategies such as 3D-CRT were designed, but these still suffer from some limitations.

The present study attempted to reduce the irradiated volume on normal tissue to increase the target irradiation dose based on two extreme quiet breathing phase CT images as templates for planning 3D-CRT. A total of 64 patients completed the $3 \mathrm{D}-\mathrm{CRT}$ treatment, and $>80 \%$ of the patients who were in Stage III or IV were successfully treated without serious complications. These results are supported by previous studies ${ }^{7,10,11}$ that have shown that a single 4D-CT study is sufficient to correctly plan 3D-CRT in most patients while minimizing the portion of normal lung being irradiated, with favorable outcomes.

The lungs possess the largest magnitude of motion in the body, and respiratory motions constitute the most serious factor affecting the accuracy of stereotactic radiotherapy for lung cancer. ${ }^{12}$ Predicting the trajectory of a target due to respiratory motion is complicated and difficult. ${ }^{13}$ Therefore, it is common to limit the range of motion in patients by using respiratory techniques, such as abdominal compression, a deep breath after a breath hold, and active breathing control, to narrow the range of motion of the targets. ${ }^{14,15}$ 
A
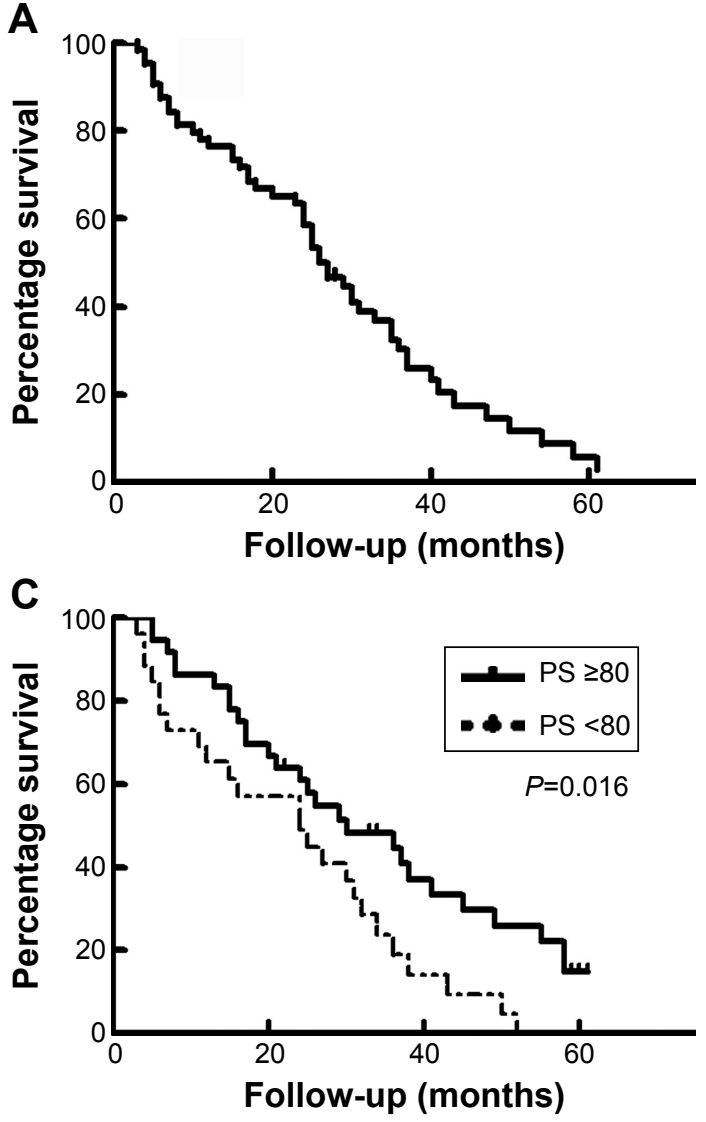

B

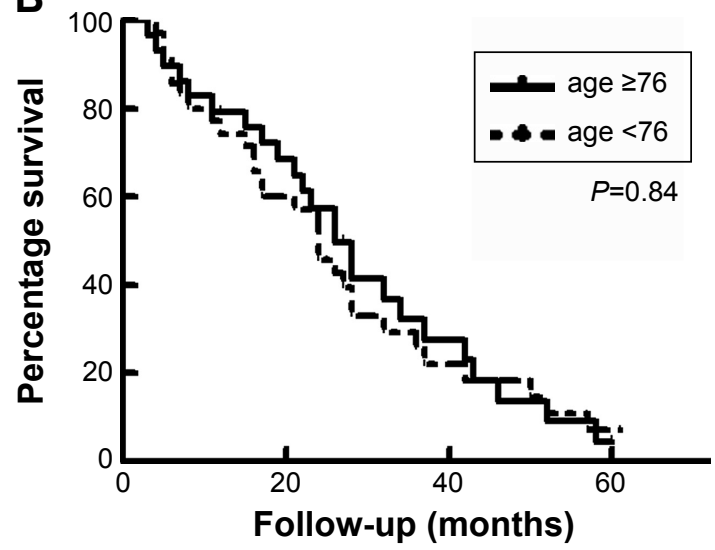

D

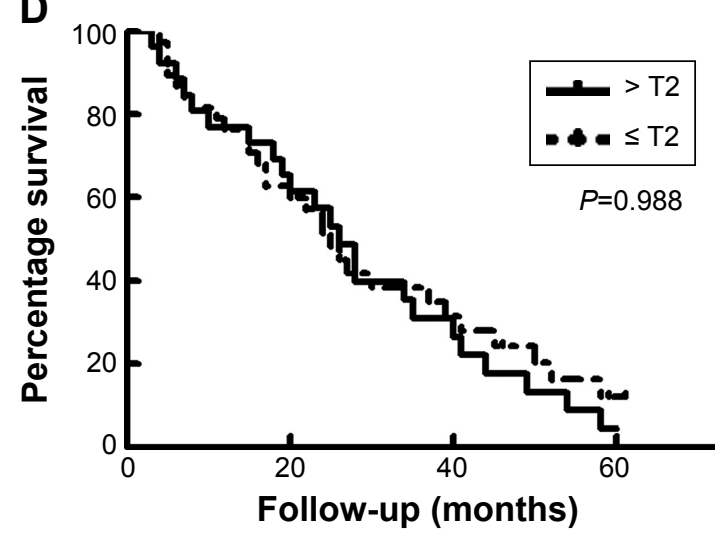

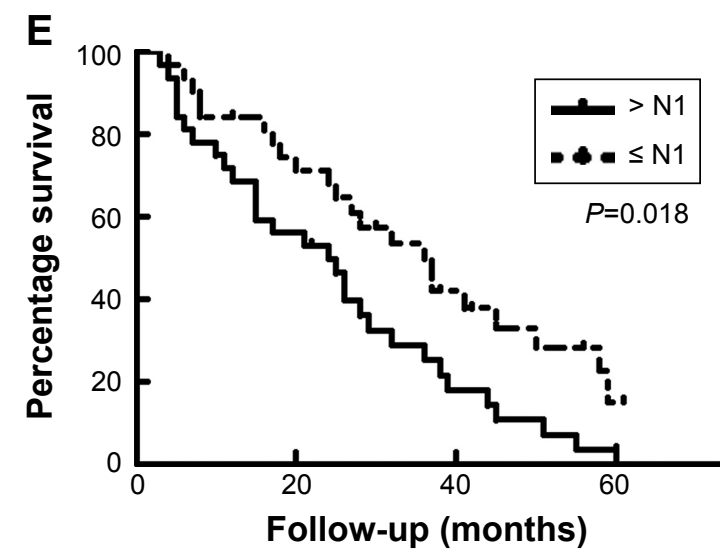

Figure 2 Overall survival curves.

Notes: (A) All patients; results according to (B) age group, (C) performance status, (D) T stage, and (E) $\mathrm{N}$ stage.

However, images obtained from CT positioning for conventional stereotactic radiotherapy are not sufficiently clear to ascertain the stage of the breathing phase, to avoid tumor leakage, to prevent errors in the setup of target encompassing, or to evaluate the magnitude of tumors in 3D. In the present study, simulations were completed with multiphase breathing CT, which resulted in the GTV of end-expiratory and end-inspiratory phases being equal to the GTV with quiet breathing; thus, changes were obtained in two extreme positions. The final GTV covering the range of tumor movement during quiet breathing was obtained by combining the two GTVs. Thus, the PTV had more substantial reduction than with the traditional method, and the high-dose-irradiated lung volume was also reduced accordingly, demonstrating that it may be safe for elderly patients who can calmly breathe during the course of treatment. 
Table 3 Cox regression analysis

\begin{tabular}{llll}
\hline & OR & 95\% Cl & \\
\cline { 3 - 4 } & & Lower & Upper \\
\hline Age & 0.986 & 0.695 & 2.673 \\
T stage & 0.973 & 0.126 & 1.287 \\
N stage & 1.212 & 1.150 & 2.074 \\
KPS & 1.259 & 1.242 & 1.667 \\
\hline
\end{tabular}

Abbreviations: $95 \% \mathrm{Cl}, 95 \%$ confidence interval; KPS, Karnofsky performance status; OR, odds ratio.

The average PTV of the 64 cases was reduced by $19.3 \%$ between the two types of radiation treatment approaches, and the average lung volume reached with a prescription dose was decreased by $21.8 \%$. However, the reduced irradiation volume did not affect the efficacy. Indeed, in the present study, the 1- to 3 -year OS rate of 64 patients was not less than that reported in the literature, and the incidence of radiation pneumonitis and esophagitis was lower than the average. ${ }^{16}$ The reason for these findings may be related to a decreased individual PTV irradiation volume. Improved OS rates may provide more adequate safeguards reflecting the quality of life of patients after treatment. Patients were given a biologically equivalent dose of $>80$ Gy because a relatively smaller irradiation volume was given, and it was indicated that we can improve the relatively safe radiation dose in elderly patients, and the risk of serious adverse events from radiation therapy alone has been demonstrated to be small.

The results of the present study strongly suggest that only the KPS score and $\mathrm{N}$ stage were independent prognostic factors, while age was not. There were six patients who survived $>5$ years; among these, four patients were aged $>80$ years, and their general states were similar to those with an earlier-stage tumor and KPS score of $\geq 80$. Muss et $\mathrm{al}^{17}$ reported 1,052 cases of NSCLC that were inoperable, and they achieved a better efficacy with platinum-based chemotherapy in elderly patients compared with young ones. Similar results were demonstrated by Langer et al. ${ }^{18}$ Therefore, the KPS score is even higher for elderly patients who can tolerate comprehensive chemotherapy treatment with radiotherapy. Thus, treatment should not be too conservative.${ }^{17}$ In particular, concurrent chemoradiotherapy for the treatment of locally advanced NSCLC has shown good potential. In general, an elderly age and poor performance status should not determine who can be treated by such a regimen for those having a higher incidence of adverse efficacy and treatment-related mortality because chemotherapy drugs can reduce normal tissue tolerance to radiation damage and interfere with radiation-damaged repair processes. ${ }^{12,19}$ Therefore, more individualized treatment approaches for elderly patients should be implemented, in which performance status may be considered for a comprehensive regimen, because a low KPS score was purely related to the safety of 3D-CRT and the variety of techniques used to reduce the PTV are conducive to maintaining quality of life.

The present study is not without limitations. Indeed, the sample size was small and was from a single center. Larger multicenter studies should be conducted to confirm our findings.

\section{Conclusion}

Without affecting the therapeutic effects, CT simulations in different respiratory phases are well-tolerated in elderly patients with NSCLC, can effectively reduce PTV, and can improve the quality of life.

\section{Disclosure}

The authors report no conflicts of interest in this work.

\section{References}

1. Jemal A, Siegel R, Xu J, Ward E. Cancer statistics, 2010. CA Cancer J Clin. 2010;60(5):277-300.

2. Aupérin A, Le Péchoux C, Rolland E, et al. Meta-analysis of concomitant versus sequential radiochemotherapy in locally advanced non-small-cell lung cancer. J Clin Oncol. 2010;28(13):2181-2190.

3. Grutters JP, Kessels AG, Pijls-Johannesma M, De Ruysscher D, Joore MA, Lambin P. Comparison of the effectiveness of radiotherapy with photons, protons and carbon-ions for non-small cell lung cancer: a meta-analysis. Radiother Oncol. 2010;95(1):32-40.

4. Herbert C, Kwa W, Nakano S, et al. Stereotactic body radiotherapy: volumetric modulated arc therapy versus $3 \mathrm{D}$ non-coplanar conformal radiotherapy for the treatment of early stage lung cancer. Technol Cancer Res Treat. 2013;12(6):511-516.

5. Oechsner M, Berndt J, Wilkens JJ, Duma MN. Respiratory-gated lung stereotactic body radiation therapy: a planning study of 3D-CRT versus VMAT. Int J Radiat Oncol Biol Phys. 2014;90(1):S904.

6. Guckenberger M, Wilbert J, Richter A, Baier K, Flentje M. Potential of adaptive radiotherapy to escalate the radiation dose in combined radiochemotherapy for locally advanced non-small cell lung cancer. Int J Radiat Oncol Biol Phys. 2011;79(3):901-908.

7. Guckenberger M, Wilbert J, Meyer J, Baier K, Richter A, Flentje M. Is a single respiratory correlated $4 \mathrm{D}$-CT study sufficient for evaluation of breathing motion? Int J Radiat Oncol Biol Phys. 2007;67(5): 1352-1359.

8. Firat S, Byhardt RW, Gore E. The effects of comorbidity and age on RTOG study enrollment in stage III non-small cell lung cancer patients who are eligible for RTOG studies. Int J Radiat Oncol Biol Phys. 2010; 78(5):1394-1399.

9. Wisnivesky JP, Halm E, Bonomi M, Powell C, Bagiella E. Effectiveness of radiation therapy for elderly patients with unresected stage I and II non-small cell lung cancer. Am J Respir Crit Care Med. 2010;181(3): 264-269.

10. Guckenberger M, Krieger T, Richter A, et al. Potential of image-guidance, gating and real-time tracking to improve accuracy in pulmonary stereotactic body radiotherapy. Radiother Oncol. 2009;91(3):288-295.

11. Wolthaus JW, Sonke JJ, van Herk M, et al. Comparison of different strategies to use four-dimensional computed tomography in treatment planning for lung cancer patients. Int J Radiat Oncol Biol Phys. 2008;70(4): $1229-1238$. 
12. Guckenberger M, Kavanagh A, Partridge M. Combining advanced radiotherapy technologies to maximize safety and tumor control probability in stage III non-small cell lung cancer. Strahlenther Onkol. 2012; 188(10):894-900.

13. Therasse P, Arbuck SG, Eisenhauer EA, et al. New guidelines to evaluate the response to treatment in solid tumors. European Organization for Research and Treatment of Cancer, National Cancer Institute of the United States, National Cancer Institute of Canada. J Natl Cancer Inst. 2000;92(3):205-216.

14. Ozhasoglu C, Murphy MJ. Issues in respiratory motion compensation during external-beam radiotherapy. Int J Radiat Oncol Biol Phys. 2002;52(5):1389-1399.

15. Kim DJ, Murray BR, Halperin R, Roa WH. Held-breath self-gating technique for radiotherapy of non-small-cell lung cancer: a feasibility study. Int J Radiat Oncol Biol Phys. 2001;49(1):43-49.
16. Saito T, Sakamoto T, Oya N. Comparison of gating around endexpiration and end-inspiration in radiotherapy for lung cancer. Radiother Oncol. 2009;93(3):430-435.

17. Muss HB. Older age - not a barrier to cancer treatment. $N$ Engl J Med. 2001;345(15):1127-1128.

18. Langer CJ, Manola J, Bernardo P, et al. Cisplatin-based therapy for elderly patients with advanced non-small-cell lung cancer: implications of Eastern Cooperative Oncology Group 5592, a randomized trial. J Natl Cancer Inst. 2002;94(3):173-181.

19. Schild SE, Stella PJ, Geyer SM, et al; North Central Cancer Treatment Group. The outcome of combined-modality therapy for stage III non-small-cell lung cancer in the elderly. J Clin Oncol. 2003;21(17): 3201-3206.

\section{Publish your work in this journal}

OncoTargets and Therapy is an international, peer-reviewed, open access journal focusing on the pathological basis of all cancers, potential targets for therapy and treatment protocols employed to improve the management of cancer patients. The journal also focuses on the impact of management programs and new therapeutic agents and protocols on

\section{Dovepress}

patient perspectives such as quality of life, adherence and satisfaction. The manuscript management system is completely online and includes a very quick and fair peer-review system, which is all easy to use. Visit http://www.dovepress.com/testimonials.php to read real quotes from published authors.

Submit your manuscript here: http://www.dovepress.com/oncotargets-and-therapy-journal 